Dieses Dokument ist eine Zweitveröffentlichung (Postprint) /

This is a self-archiving document (accepted version):

T. Mikolajick, H. Mulaosmanovic, M. Hoffmann, B. Max, T. Mittmann, U. Schroeder, S. Slesazeck

Variants of Ferroelectric Hafnium Oxide based Nonvolatile Memories

Erstveröffentlichung in / First published in:

Device Research Conference (DRC). Ann Arbor, 2019. IEEE, S. 207-208. ISBN 978-1-7281-2112-3

DOI: https://doi.org/10.1109/DRC46940.2019.9046455

Diese Version ist verfügbar / This version is available on:

https://nbn-resolving.org/urn:nbn:de:bsz:14-qucosa2-775772 


\title{
Variants of Ferroelectric Hafnium Oxide based Nonvolatile Memories
}

\author{
T. Mikolajick ${ }^{1,2}$, H. Mulaosmanovic ${ }^{1}$, M. Hoffmann ${ }^{1}$, B.Max $^{2}$, T. Mittmann ${ }^{1}$, \\ U. Schroeder ${ }^{1}$, and S. Slesazeck ${ }^{1}$ \\ ${ }^{1}$ NaMLab gGmbH, Noethnitzer Str. 64, Dresden, D-01187, Germany \\ ${ }^{2}$ Chair of Nanoelectronic Materials, TU Dresden, Dresden, D-01069, Germany \\ Email:Thomas.mikolajick@namlab.com /Phone: (+49)351-212499020
}

\section{Introduction}

Ferroelectricity is very attractive for nonvolatile memories since it allows non-volatility paired with a field driven switching mechanism enabling a very low-power write operation. Non-volatile memories based on ferroelectric leadzirconium-titanate (PZT) (see fig. 1a) are available on the market for more than a quarter of a century now [1]. Yet they are limited to niche applications due to the compatibility issues of the ferroelectric material with CMOS processes and the associated limited scalability [2]. The discovery of ferroelectricity in doped hafnium oxide has revived the activities towards a variety of scalable ferroelectric nonvolatile memory devices [3].

\section{Ferroelectricity in Hafnium Oxide}

Under certain processing conditions a non-centrosymmetric orthorhombic phase can be stabilized in hafnium oxide thin films [4]. In such a crystal structure, the oxygen ions can switch between two stable positions. The schematic of a unit cell of an orthorhombic hafnium oxide is shown in fig. $1 \mathrm{~b}$ in comparison to the unit cell of a perovskite like PZT (fig. 1a) were the cation on the $\mathrm{B}$ lattice site of the $\mathrm{ABO}_{3}$ structure is assumed to be responsible for ferroelectric switching.

\section{Advancements in Ferroelectric Memory Cell Concepts}

There are three different ways to read out the ferroelectric polarization and construct a memory cell (fig.2). First the charge switched during the polarization switching in a capacitor can be directly detected which leads to the classical ferroelectric random access memory (FeRAM, see fig. 2a) [5]. Second, the ferroelectric can be integrated into the gate stack of a MOS transistor leading to a ferroelectric field effect transistor (FeFET, see fig. 2b) [5]. Finally, in very thin ferroelectric layers that allow tunneling, the magnitude of the tunneling current depends on the polarization direction leading to a ferroelectric tunnel junction (FTJ, see fig. 2c) [6]. In the capacitor based FeRAM, the material properties of the ferroelectric are directly reflected. The coercive field of hafnium oxide based ferroelectrics is in the region of $1-2 \mathrm{MV} / \mathrm{cm}$ leading to a high electrical field stress during endurance cycling and degradation of the ferroelectric. In particular oxygen vacancies play a crucial role in the optimization since they seem to support the stabilization of the orthorhombic phase [7] while at the same time they are detrimental for the endurance [8].

FeFET devices have been successfully integrated into $28 \mathrm{~nm}$ [9] and $22 \mathrm{~nm}$ [10] technologies in the last years. The unavoidable interface oxide between the channel and the ferroelectric and the sensitivity of the MOSFET to any trapped charge makes cycling degradation even more severe [11]. Increasing the thickness of the ferroelectric layer helps to reduce the stress on the tunneling oxide during cycling [12]. A self-heating technique can be utilized to enhance cycling by annealing the cycling damage during device operation [13]. Furthermore, with an additional metal electrode the area ratio between the MOS capacitor and the ferroelectric capacitor can be optimized [11]. A basic limitation of the classical FeRAM cell shown in fig. 2a is the necessary capacitor area for stable sensing. 3D capacitors are necessary when scaling below 90nm [14]. When connecting the capacitor to the gate of a MOSFET, the amplification of the MOSFET can be utilized to drastically reduce the necessary capacitor area. However, a second transistor is required to reset the potential on the internal node (see fig. 3a). Such a cell was recently fabricated using a $28 \mathrm{~nm}$ technology were the gate capacitor of a FeFET was used as a storage capacitor.[15] (fig. 3b). Fig. 4 shows the measurement results. The interface oxide in series with the ferroelectric in the FeFET can be also utilized as a double layer tunnel junction [16]. The respective operation in this configuration mode is illustrated in fig. 5. The talk will start with a summary on the current understanding of the optimization of ferroelectricity in hafnium oxide and explain the implications for applications in FeRAMs, FeFETs and FTJs in detail.

\section{References}

[1] D. Bondurant, Ferroelectrics 112, 273-282 (1990) [2] C.-U. Pinnow and T. Mikolajick, J. Electrochem. Soc. 151, K13-K19 (2004)

[3] T. S. Böscke et al., Appl. Phys. Lett., 99: 102903 (2011) [4] M. Hoffmann et al, Journal of Applied Physics 118, 072006 (2015)

[5] T. Mikolajick et al., MRS Bulletin 43, 340 (2018) [6] E.Y. Tsymbal and H. Kohlstedt, Science, 313 (5784), 181-183, 2006 [7] T. Mittmann, Advanced Materials Interfaces (2019) [8] Russia [9] M. Trentzsch et al., IEDM 2016, pp. 11.5.1-11.5 (2016) [10] S. Dünkel et al., IEDM 2017, pp. 19.7.1-19.7.4 (2017) [11] J. Mueller et al., 16th NVMTS 2016, pp. 1-7 (2016) [12] H Mulaosmanovic et al., IEEE Electron Device Letters, in review [13] H Mulaosmanovic et al., IEEE Electron Device Letters 40 (2), 216-219 (2019) [14] K. Maruyama et al., M. Kondo, S.K. Singh, H. Ishiwara, Fujitsu Sci. Tech. J 43, 502-507 (2007) [15] S. Slesazeck et al., IMW (2019) [16] B. Max, ESSDERC 2018, 142-145, (2018) 


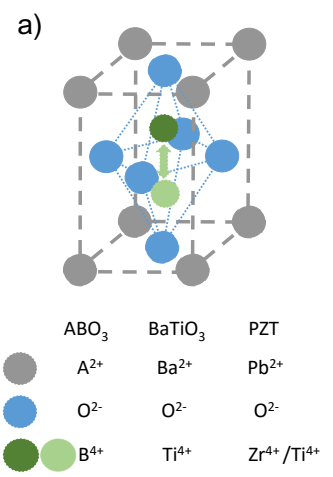

Fig. 1. Crystal structure of a) perovskite and b) orthorhombic hafnium oxide. The possible switching of the cation in a) and the oxygen ions in b) is indicated by the green arrows b)

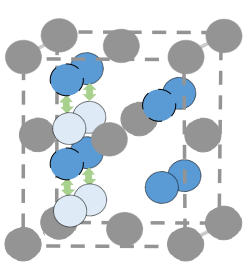

$\mathrm{HfO}_{2}$

$\mathrm{Hf}^{4+}$

$\bigcirc \mathrm{O}^{2}$ a) $1 \mathrm{~T} / 1 \mathrm{C}$

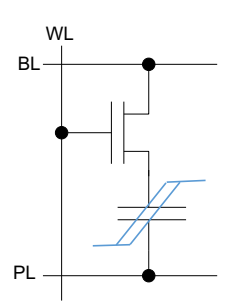

b) FeFET

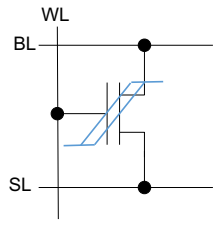

c) FTJ

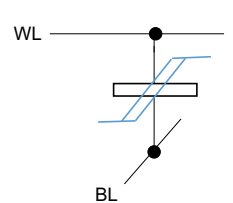

Fig. 2. Three different cell concepts for ferroelectric memories. a) Classical ferroelectric random access memory (FeRAM) cell with many similarities to a DRAM cell, b) Ferroelectric field effect transistor (FeFET) and c) Ferroelectric tunnel junction (FTJ) in a cross-bar without selector device

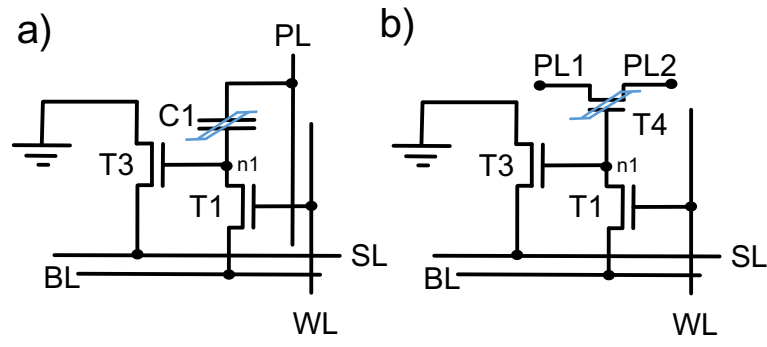

Fig. 3. $2 \mathrm{~T} / 1 \mathrm{C}$ gain FeRAM cell. a) Basic concept. The read transistors $\mathrm{T} 3$ amplifies the voltage on the internal node 1 making very small switched charge detectable. b) Experimentally realized version were the gate capacitor of a FeFET device is used as the storage capacitor.

a)

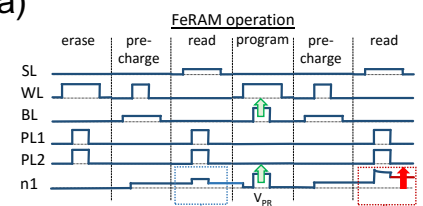

b)

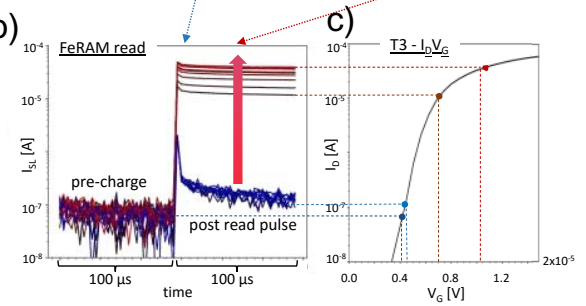

Fig. 4. Experimental results from the $2 \mathrm{~T} / 1 \mathrm{C}$ cell of fig. 3. a) applied and measured signals, b) voltage measured on SL during reading of ' 1 ' (blue) and ' 0 ' (red), c) I-V curve of $\mathrm{T} 3$ showing the different operation points. a)

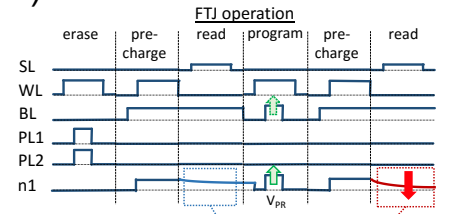

b)

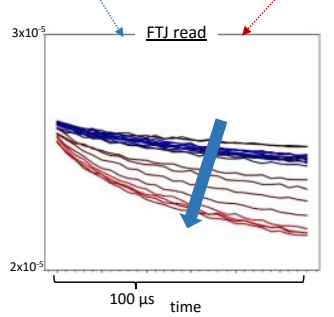

Fig. 5. Experimental results from the $2 \mathrm{~T} / 1 \mathrm{C}$ cell of fig. 3 when reading in FTJ mode a) applied and measured signals, b) voltage measured on SL during reading of ' 1 ' (blue) and ' 0 ' (red). 